\title{
The research about editing system of performance information for player piano - Make inferences about both handed musical composition by using DP matching system -
}

\author{
Ryo Kinoshita \\ Department of Intelligent and Control Systems, Kyushu Institute of Technology, \\ 680-4, kawazu, Iizuka-City, Fukuoka, 820-8502, Japan* \\ Eiji Hayashi \\ Department of Intelligent and Control Systems, Kyushu Institute of Technology, Hayashi Lab, \\ 680-4, kawazu, Iizuka-City, Fukuoka,820-8502, Japan \\ E-mail:kinoshita@mmcs.mse.kyutech.jp,haya@mse.kyutech.ac.jp \\ http://www.kyutech.ac.jp/
}

\begin{abstract}
We have developed a system that allows a piano to perform automatically. In order to play music in the manner of a live pianist, we must add expression to the piano's performance. Therefore, we have developed an interactive musical editing system that utilizes a database to edit music more efficiently.

Keywords: Automatic Piano, Knowledge Database, Computer Music, Music Interface
\end{abstract}

\section{Introduction}

We have developed a system that allows a piano to perform automatically. In this system, 90 actuators have been installed on the keys and pedals of a grand piano. These actuators execute key strokes and pedal movements to govern the piano's performance, e.g., "Fig. 1.(Continued)" $[1,2]$.

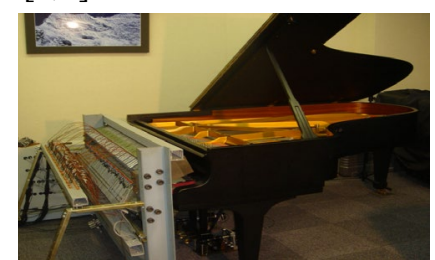

Fig. 1. The automatic piano

Our research focuses on giving player piano performance with human-like skill and expression. Playing the piano expressive by player piano, it is necessary to adjust the volume, length, and timing of music. We aimed to develop a system that, like a skilled pianist, can perform even the first musical score based on information related to previous skills and experience. So, we developed a system that automatically estimates the performance expression of unedited music using edited performance data and score data. In this paper, we described a phrase search using Dynamic Programming (DP) matching and a method for selecting an optimal phrase, how to infer parameters of notes, evaluation of an inferred entire song played by both-handed.

\section{Editing Support System}

\subsection{Performance Information}

The automatic piano that we have developed uses a music data structure that is similar to Musical Instrument Digital Interface (MIDI). This system edits four 
parameters involved in producing a tone: "Velo" (velocity), "Gate", "Step" and "Time". "Velo" is the dynamics, given by the value of 1-127. "Gate" is the duration of the note in milliseconds. "Step" is the interval of time between notes, and it also exhibits tempo. "Time" is the time since the sound started.

\subsection{How to Make the Data for Player Piano}

We show the structure of the edit system how to make the data for player piano in "fig. 2. (Continued)". The system extract features of a pianist from the music data that the pianist has played. Next, the system infers performance data from the features and score information on a music that the pianist has not played.

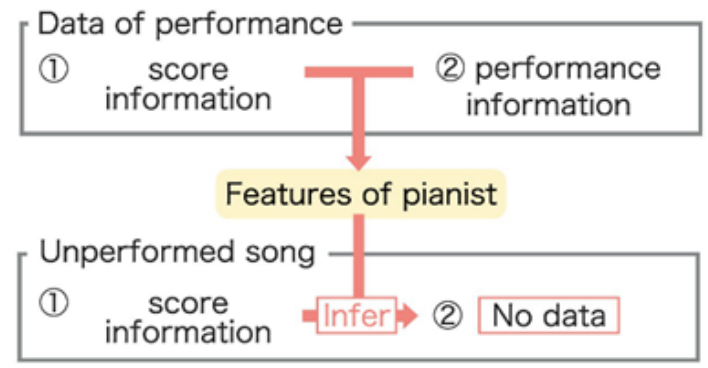

Fig. 2. The structure of the edit system

\subsection{Search System}

As a result of the analysis, it was found that phrases of the same pattern existing in the same tune are performed in a similar expression (Fig. 3.). This time, we use DP matching to search for similar phrases for arbitrarily determined search phrase.

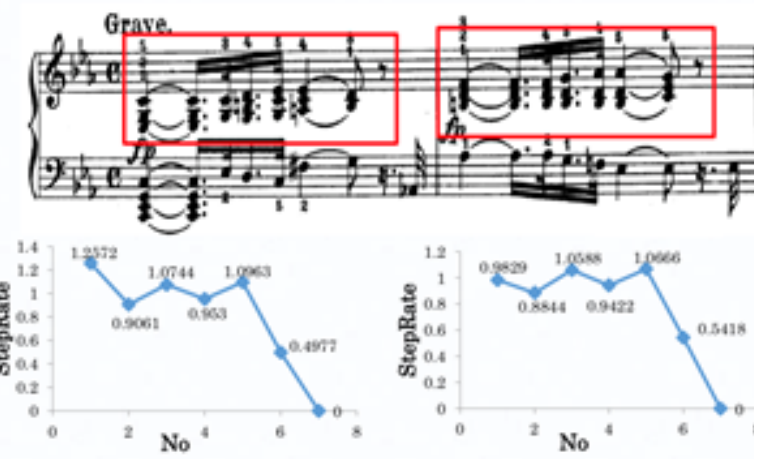

Fig. 3. The discovery about the relation of same tunes and similar expression.

DP matching has a feature that can calculate the similarity between two words that are different in a number of characters from each other.

\subsection{Select System}

After done the Search system, there are a lot of similar phrases which has the same DP matching points. Similar phrases are phrases we found by doing DP matching from music data. Objective phrase is phrase we want to inference in this time. Then, we need to select the optimal phrases for objective phrases by doing a select system. In this system, five indicators were used from the viewpoint of music theory: Dynamic symbols, Beats and Steps, Similarity in change of interval, Dynamic symbols and Velo, and Musical forms.

\subsubsection{Dynamic symbols}

If the Dynamic symbols are different between similar phrase and objective phrase, the performance is affected even if the phrase is the same. Then, the search phrase and the similar phrase that matches the dynamics on the score are preferentially selected.

\subsubsection{Beat and step}

Based on the musical grammar, it is known that strong beats are closely related to Step [3]. In places considered to be strong beats, similar phrases are selected using the property that the Step value is larger than in other places. The position of the strong beat depends on the rhythms.

\subsubsection{Similarity in change of interval}

If the phrases have similar change of interval, it is assumed that the performance expression is also similar. Therefore, we select a phrase with more similarity in change of interval.

\subsubsection{Dynamic symbols and Velo}

There is likely to be a trend in Velo value depending on dynamic symbols. Therefore, we examine the range of Velo for each dynamic symbol from performance data, and select a phrase which Velo value is correspond to search phrase's dynamic symbol.

\subsubsection{Musical forms}

If the Select system in 2.4.1.-2.4.4. does not narrow down the number of similar phrases, the selection is performed according to the Musical Forms. Musical Forms is a music format such as the Rond Forms or the Sonata Forms. For example, if different songs were in the sonata 
format, it was predicted that the exposition part had a presentation part feature, the development part had a development part feature, and each part had a unique feature. We decide same part has similar performance.

\subsubsection{Case of Left Hand}

Left hand phrases can divide four categories: Main theme, Broken chord, Single, Chord. When select left hand's phrase, select from same category. If similar phrase does not exist in same category, the system selects optimal phrase from other categories.

\subsection{Infer System}

If we use the optimal phrase's expression to search phrase, it will be unnatural expression. Hence, this system infers appropriate Step, Velo, Gate value for the search phrase from the optimal phrase.

\subsubsection{Investigation Required for Inference}

For appropriate inference, it need investigation from performance data. This year, we use four songs, and its title is shown in "Table 1". All of them are made by W.A.Mozart and Pianist is Maria Joao Pires.

Table 1. The studied title of musical compositions.

\begin{tabular}{|c|c|}
\hline & Title of musical compositions. \\
\hline (1) & $\begin{array}{c}\text { Piano Sonata No.11 in A major, K.311 3rd } \\
\text { movement "Turkish March" }\end{array}$ \\
\hline (2) & $\begin{array}{c}\text { Piano Sonata No.15 in C major, K.545 1st } \\
\text { movement "Allegro" }\end{array}$ \\
\hline (3) & $\begin{array}{c}\text { Piano Sonata No.15 in C major, K.545 2nd } \\
\text { movement "Andante" }\end{array}$ \\
\hline (4) & $\begin{array}{c}\text { Piano Sonata No.15 in C major, K.545 3rd } \\
\text { movement "Rondo" }\end{array}$ \\
\hline
\end{tabular}

\subsubsection{Inference of Step}

The investigation of tempo and normalization factor are shown in "Table 2" by using music data in "Table 1". Normalization Factor is s number that "All Tempo Average" divide "Tempo Average".

Table 2. Investigation about tempo and Normalization Factor

\begin{tabular}{|c|c|c|c|c|}
\hline Music Number & $(1)$ & 2 & $(3)$ & $(4)$ \\
\hline Tempo Avg. & 0.80 & 0.82 & 1.00 & 0.92 \\
\hline All Tempo Avg. & \multicolumn{5}{|c|}{0.89} \\
\hline Normalization Factor & 1.11 & 1.07 & 0.89 & 0.96 \\
\hline
\end{tabular}

The inference equation for Step is "Eq. (1)". "PStep" represent provisional Step value which calculate by optimal phrase and "NF" represent normalization factor.

$$
\text { Step }=\text { PStep } \times \text { NF } \times \text { All Tempo Avg. }
$$

In the case of left-hand, adjust the timing using the results of right-had inference when the note is same timing in musical score.

\subsubsection{Inference of Velo}

The investigation of the ranges and average value about Velo are shown in "Table 3" by using music data in "Table 1".

Table 3. Velo's average value for each Dynamic symbol

\begin{tabular}{|c|c|c|}
\hline $\begin{array}{c}\text { Dynamic } \\
\text { symbol }\end{array}$ & $\begin{array}{c}\text { Avg. } \\
\text { (right-hand) }\end{array}$ & $\begin{array}{c}\text { Avg. } \\
\text { (left-hand) }\end{array}$ \\
\hline $\mathrm{p}$ & 64 & 44 \\
\hline $\mathrm{mf}$ & 62 & 36 \\
\hline $\mathrm{f}$ & 64 & 45 \\
\hline
\end{tabular}

When the system infers Velo's value, it uses similar interval phrase. Similar interval phrase is the phrase with the highest similarity of interval changes among similar phrases.

The inference equations for Velo are "Eq. (2)" and "Eq. (3)", "Eq. (4)". " $n$ " represents the number of notes in objective phrase and "SVelo" represents similar interval phrase's value of Velo. Eq. (2) is used when the first note in the phrase and the dynamic symbol is different from the previous note. Depending on the results of DP matching, the search phrase may have to be split. In this case, the first note of split phrase is calculated by Eq. (3) except case of Eq (2). Other notes are calculated by Eq (4).

$$
\begin{gathered}
\operatorname{Velo}(\mathrm{n})=\text { average in dynamics symbol } \\
\operatorname{Velo}(\mathrm{n})=\operatorname{Velo}(\mathrm{n}-1) \\
\operatorname{Velo}(\mathrm{n})=\operatorname{Velo}(\mathrm{n}-1)-\text { SVelo's value }
\end{gathered}
$$

\subsubsection{Inference of Gate}

The investigation of factors for each musical symbol that gives a change in the length of a note are shown in "Table 4 " by using music data in "Table 1 ". 
Table 4. Factor for each musical symbol

\begin{tabular}{|c|c|c|c|}
\hline Musical symbols & staccato & No symbol & slur \\
\hline Factor & 0.4 & 0.9 & 1 \\
\hline
\end{tabular}

The inference equation for Gate is "Eq. (5)". Gate $=$ inferred Step $\times$ Factor

\section{Inference Experiment}

An experiment was conducted to compare the music reproduced using the editing support system with the performance of the pianist. The target song is Mozart Piano Sonata No.11 in A major, K.311 1st movement "Theme". Some of the inference result are shown as graphs. "Fig. 4" is about right-handed and "Fig. 5"is lefthanded Step. "Fig. 6" is about right-handed Gate. "Fig. 7" is about right-handed Velo.

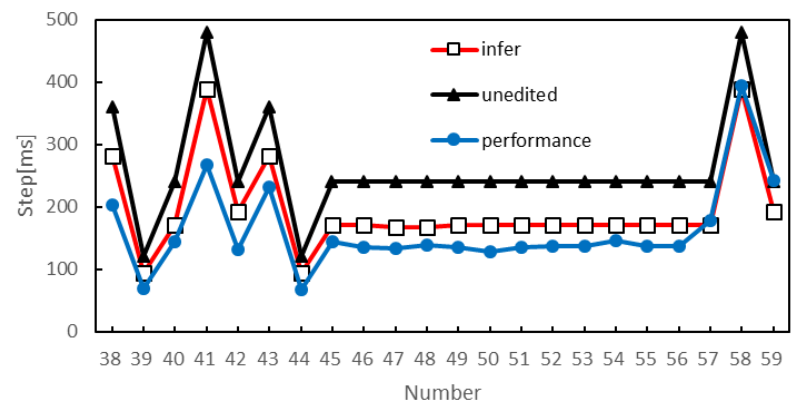

Fig. 4 Inference result about right-handed Step

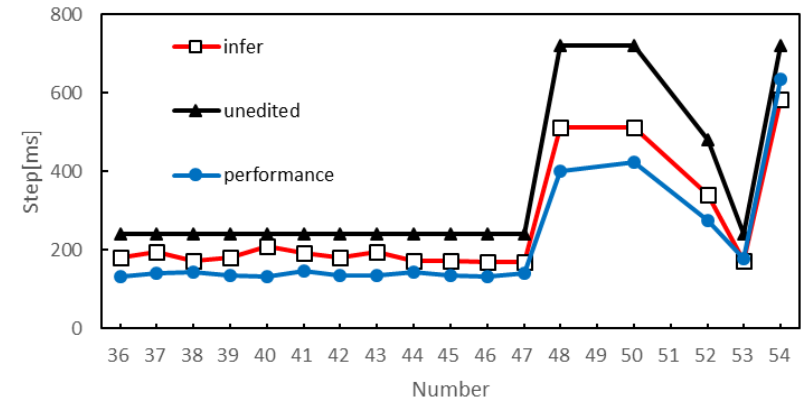

Fig. 5. Inference result about left-handed Step

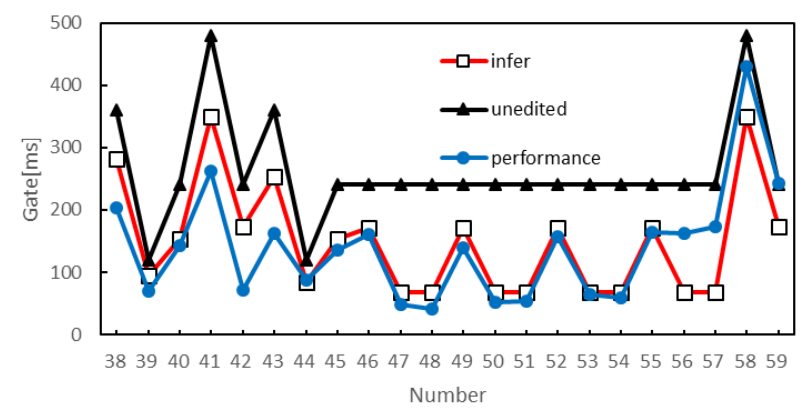

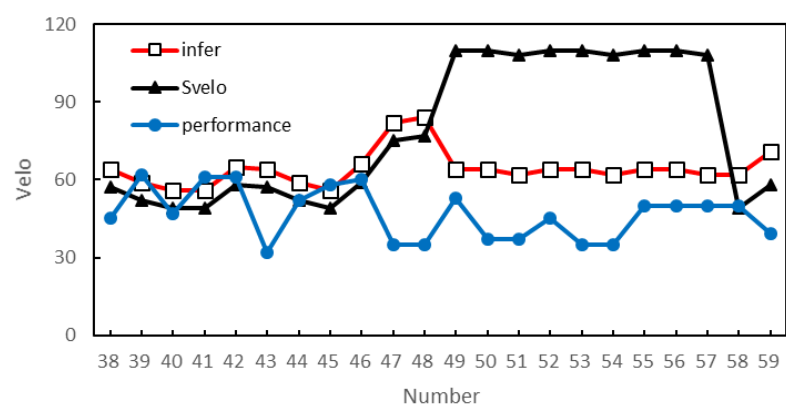

Fig. 7. Inference result about right-handed Velo

\section{Consideration}

We can confirm that inferred Step value is closer to the pianist's performance than unedited data. It is common to both hands and the same could be seen in Gate. Thus, the results suggest that infer system about Step and Gate are effective. However, inferred Velo's value is not similar to performance data. Therefore, infer system about Velo is need to improve.

\section{Conclusion}

This year, we introduced a left-handed inference system and Gate's inference system in the editing system of performance information. The inference experiment showed that we were able to infer a performance expression in an unperformed song from data of performance.

Editing system of performance information is infer a phrase from similar phrase in other songs. And, when the same phrase is repeated, the infer result is exactly same. However, pianists should subtly change in inflection and timing for the same phrase. Furthermore, the current system can't infer performance information when the score doesn't have dynamic symbols. In order to solve these problems, we need to develop a new versatile system that adds other methods to current system.

\section{References}

1. Y. Isomichi and N. Ogawa, Pattern Matching by Using Dynamic Progaming, 1975, Vol 16, No.1, pp.15-22.

2. 2. E.Hayashi, M.Yamane and H.Mori, Development of Moving Coil Actuator for an Automatic Piano, Inrwenational Journal of Japan Society for Precision Engineering, Vol.28 No.2, 1994, pp.164-169.

3. Shimaoka Jo, Analize about Harmony and Musical style, ONGAKU NO TOMO SHA CORP, 1964.

Fig. 6. Inference result about right-handed Gate

(C) The 2021 International Conference on Artificial Life and Robotics (ICAROB2021), January 21 to 24, 2021 